\title{
Visfatin/NAMPT is unrelated to nonalcoholic fatty liver histology but correlates with liver recovery after partial liver resection: Data from a pilot study
}

\author{
Lock JF ${ }^{1}$, Sponholz $\mathrm{C}^{2}$, Hoppe $\mathrm{S}^{3}$, Döcke $\mathrm{S}^{4}$, Rieger $\mathrm{A}^{5}$, Birkenfeld AL ${ }^{6}$, Pfeiffer $\mathrm{AFH}^{4,7}$, Stockmann $\mathrm{M}^{3}$, Bauer $\mathrm{M}^{2,8}$ and Von Loeffelholz $\mathrm{C}^{2,8 *}$ \\ ${ }^{1}$ Department of General-, Visceral-, Vascular- and Paediatric Surgery, University Hospital of Wuerzburg, Wuerzburg, Germany \\ ${ }^{2}$ Department of Anaesthesiology and Intensive Care, Jena University Hospital, Jena, Germany \\ ${ }^{3}$ Department of General, Visceral and Transplantation Surgery, Charité-Universitätsmedizin, Berlin, Germany \\ ${ }^{4}$ Department of Clinical Nutrition, German Institute of Human Nutrition Potsdam-Rehbruecke, Nuthetal, Germany \\ ${ }^{5}$ Institute of Pathology, Charité-Universitätsmedizin, Berlin, Germany \\ ${ }^{6}$ Section of Metabolic and Vascular Medicine, Medical Clinic III, University Hospital Carl Gustav Carus, Dresden, Germany and Competence Center for Metabolic \\ Vascular Medicine Prof. Hanefeld, GWT- TU Dresden, Dresden, Germany and Section of Diabetes and Nutritional Sciences, King's College London, London, UK \\ ${ }^{7}$ Department of Endocrinology, Diabetes, and Nutrition, Charité - Universitätsmedizin, Berlin, Germany \\ ${ }^{8}$ Integrated Research and Treatment Center, Center for Sepsis Control and Care (CSCC), Friedrich Schiller University, Jena, Germany
}

\begin{abstract}
Background/Aims: Nonalcoholic fatty liver disease (NAFLD) is the worldwide most common liver disease and therefore frequently observed in patients undergoing hepatic surgery. Etiologically NAFLD relates to insulin resistance and can progress to fibro-inflammatory nonalcoholic steatohepatitis (NASH). This study investigated associations of hepatic mRNA and preoperative serum levels of the Nicotinamide-phosphoribosyltransferase (NAMPT) visfatin with NAFLD histology, markers of insulin resistance, inflammation and postoperative liver recovery.

Methods: NAFLD histology was evaluated in 44 subjects undergoing liver surgery. Fasting blood samples and liver biopsies were harvested. Clinical chemistry and homeostasis model of insulin resistance (HOMA-IR) were evaluated. Circulating visfatin/NAMPT was measured by enzyme linked-immunosorbent assay, mRNA by RT-PCR. Postoperative liver recovery was studied in a subset of subjects using the LiMAx-method.

Results: Visfatin/NAMPT mRNA and serum levels showed no associations with hepatic steatosis, fibro-inflammation, ballooning degeneration or insulin resistance $(P>0.05$, resp.). Circulating visfatin/NAMPT correlated with ferritine $(\mathrm{r}=0.325 ; P=0.032)$ and serum iron $(\mathrm{r}=-0.342 ; P=0.023)$ as surrogates of hepatic oxidative stress, liver mRNA with white blood cell count $(\mathrm{r}=0.357 ; P=0.062)$ and hepatic CRP $\mathrm{mRNA}(\mathrm{r}=0.346 ; P=0.061)$. Moreover, circulating visfatin/NAMPT was related to mean postoperative LiMAx liver recovery $(\mathrm{r}=0.480 ; P=0.044)$ and subjects reaching physiological liver function capacity within ten days after surgery had elevated preoperative serum visfatin/NAMPT $(P=0.014)$.
\end{abstract}

Conclusions: Visfatin/NAMPT is related to markers of oxidative stress, inflammation and liver recovery after hepatic surgery, but not to NAFLD histology or insulin resistance.

Abbreviations: ELISA: enzyme-linked immunosorbent assay; HOMA-IR: homeostasis model assessment of insulin resistance; NAS: NAFLD activity score; NAFLD: nonalcoholic fatty liver disease; NAMPT: Nicotinamide-phosphoribosyltransferase; NASH: nonalcoholic steatohepatitis

\section{Introduction}

Consistent with the prevalence of obesity nonalcoholic fatty liver disease (NAFLD) affects up to $30 \%$ of the general population [1]. NAFLD is etiologically related to insulin resistance and typically categorized in hepatocellular steatosis and nonalcoholic steatohepatitis (NASH) [1]. Approximately one third of NAFLD cases progress to NASH characterized by concurrent liver steatosis, fibrosis and necroinflammation, feasibly resulting in liver cirrhosis and hepatocellular carcinoma (HCC) [1-3]. Correspondingly, NASH is nowadays a common comorbidity in perioperative patients and associated with the incidence of postoperative complications [4-6]. The Nicotinamidephosphoribosyltransferase (NAMPT) visfatin has important cellular function due to synthesizing nicotinamide-mononucleotide and was initially described as adipokine with insulin-mimetic effects [7]. Subsequent studies evidenced that visfatin/NAMPT can be expressed by various cells including hepatocytes and macrophages, while the insulin-like effect of visfatin/NAMPT has been challenged and rather

${ }^{\star}$ Correspondence to: Christian von Loeffelholz, Department of Anaesthesiology and Intensive Care, Friedrich Schiller University of Jena, Jena Germany, Am Klinikum 1, 07749 Jena, Germany, Tel: +49-3641/9322225; Fax: +493641/323102; E-mail: Christian.von_loeffelholz@med.uni-jena.de

Key words: insulin resistance, limax, nafld, partial hepatectomy, visfatin/NAMPT Received: June 05, 2018; Accepted: June 18, 2018; Published: June 22, 2018 
Lock JF (2018) Visfatin/NAMPT is unrelated to nonalcoholic fatty liver histology but correlates with liver recovery after partial liver resection: Data from a pilot study

proinflammatory characteristics were proclaimed [8,9]. The role of visfatin/NAMPT in human NAFLD remains controversial [10-17] yet recent data indicate potential protective effects in hepatocytes [12]. We intended to examine potential relationships of visfatin/NAMPT with NAFLD histology and insulin resistance in patients undergoing liver surgery. Moreover, in the same study group we postoperatively analysed liver function to evaluate possible associations with visfatin/NAMPT.

\section{Methods}

\section{Experimental design}

We evaluated associations of hepatic visfatin/NAMPT mRNA as measured in intraoperatively harvested liver specimens, and of circulating visfatin/NAMPT with NAFLD histology and insulin resistance in patients undergoing elective liver surgery due to nonmalignant or malignant indication. Furthermore, visfatin/NAMPT is expressed in hepatocytes and associated with inflammation and antiapoptosis. We therefore evaluated postoperative functional liver recovery in a subset by using the LiMAx method, to assess potential associations with visfatin/NAMPT.

\section{Ethics and study subjects}

Subjects were included in previous studies and had to meet predefined inclusion criteria $[18,19]$. The study was approved by the ethical committee of the Charité University Hospital and conforms to the provisions of the Declaration of Helsinki.

\section{Clinical characterization and assays}

Medical history, body mass index (BMI), non-invasive arterial blood pressure, anthropometry and physical examination were evaluated. Fasting blood samples were drawn and clinical chemistry was analysed. Insulin resistance was assessed by the homeostasis model assessment of insulin resistance (HOMA-IR) [20]. Full-length visfatin/ NAMPT was measured using an enzyme-linked immunosorbent assay (ELISA; AdipoGen Inc., Korea; mean intraassay CV: $<6.0 \%$; interassay $\mathrm{CV}:<10 \%)$.

\section{Wedge biopsy}

Surgery was performed in general balanced anaesthesia [18], liver samples were taken following a specified protocol [19]. A blinded pathologist confirmed samples to be non-pathologic except for NAFLD. NAFLD histopathology was evaluated and NASH was diagnosed according to the presence of at least two of the following criteria, 1) NAFLD activity score $\geq 5$; 2) presence of ballooning degeneration; 3) fibrosis score $\geq 2[21,22]$. Subjects free from hepatic steatosis or suffering from minor NAFLD criteria were considered as non-NASH reference group.

\section{LiMAx}

Using ${ }^{13} \mathrm{C}$-labeled methacetin that is metabolized to ${ }^{13} \mathrm{CO}_{2}$ by cytochrome P450 1A2 the LiMAx method evaluates maximal enzymatic liver function capacity by a non-invasive breath test [23]. The physiological cut-off value is $>315 \mu \mathrm{g} / \mathrm{kg} / \mathrm{h}$ [23]. Early liver regeneration is normally achieved within ten days after surgery [24]. We performed a basal LiMAx test before surgery and subsequent analyses at days 1, 3, 5 and 10.

\section{Real-time polymerase chain reaction}

Analysis was performed as previously reported [18]. Primer sequences were 5'-TGACACTGGCAAAACAATGCA-
3'/5'-GGTCCTTTTCACCAGCAAGCT-3' for HPRT [18] and 5'-GATCCCAACAAAAGGTCC A-3'/5'-ATGATGTGCTGCTTCCA-3' for visfatin/NAMPT [25].

\section{Statistics}

Data are means \pm SE. Using SPSS 16.0 (Chicago, USA) normal distribution was verified and logarithmic transformation performed if necessary. Spearman's rank or Pearson's coefficient were applied for correlation analysis, Mann-Whitney $U$ test was used for approving significant effects. Significance was defined as two-sided $P<0.05$.

\section{Results}

\section{Subjects}

44 subjects were included, 25\% were categorized as NASH and $75 \%$ as not NASH (Table 1). NASH patients had higher incidence of HCC, increased BMI and waist circumference, HOMA-IR, serum triglycerides and NAFLD scorings $(P<0.05$, resp.; Table 1$)$. NASH was accompanied by elevated alanine-transaminase and ferritine levels. Basal LiMAx values varied within the expected physiological range of $>$ $315 \mu \mathrm{g} / \mathrm{kg} / \mathrm{h}$, but NASH subjects had reduced levels $(P>0.05$; Table 1$)$.

\section{NAFLD histology, inflammation, markers of insulin} resistance

By addressing NAFLD we observed no differences regarding circulating visfatin/NAMPT in liver steatosis vs. no steatosis $(3.3 \pm$ 0.7 vs. $2.6 \pm 0.8 ; P=0.54$ ), in lobular inflammation vs. absent lobular inflammation $(3.0 \pm 0.9$ vs. $3.1 \pm 0.7 ; P=0.73)$, in present vs. absent hepatocellular ballooning degeneration $(2.4 \pm 0.4$ vs. $3.2 \pm 0.6 ; P=0.51)$ or in liver fibrosis vs. absence of fibrosis ( $3.2 \pm 0.7$ vs. $2.8 \pm 0.7 ; P=0.70)$. Moreover, we evaluated visfatin/NAMPT by stratifying our subjects

Table 1. Study subject characteristics

\begin{tabular}{|c|c|c|c|}
\hline & Not NASH & NASH & $P$-value \\
\hline $\mathrm{n}(\%$ male $)$ & $33(39)$ & $11(36)$ & - \\
\hline Age (y) & $58 \pm 3$ & $60 \pm 3$ & 0.85 \\
\hline Body mass index $\left(\mathrm{kg} / \mathrm{m}^{2}\right)$ & $24.4 \pm 0.6$ & $32.1 \pm 2.4$ & $<0.001$ \\
\hline Waist circumference $(\mathrm{cm})$ & $91.1 \pm 2.4$ & $108.3 \pm 4.6$ & 0.002 \\
\hline Systolic blood pressure (mm Hg) & $131 \pm 4$ & $135 \pm 5.0$ & 0.29 \\
\hline Diastolic blood pressure $(\mathrm{mm} \mathrm{Hg})$ & $71 \pm 2$ & $76 \pm 3$ & 0.20 \\
\hline C-reactive protein (mg/dl) & $1.7 \pm 0.6$ & $1.6 \pm 0.7$ & 0.24 \\
\hline White blood cell count $\left(\mathrm{x} 10^{3} / \mathrm{mm}^{3}\right)$ & $6.7 \pm 0.4$ & $6.9 \pm 0.6$ & 0.75 \\
\hline HbA1c (\%) & $5.5 \pm 0.1$ & $6.1 \pm 0.5$ & 0.29 \\
\hline HOMA-IR & $2.1 \pm 0.4$ & $5.3 \pm 1.4$ & 0.008 \\
\hline Serum triglycerides $(\mathrm{mg} / \mathrm{dl})$ & $109.0 \pm 8.7$ & $159.6 \pm 17.7$ & 0.005 \\
\hline Bilirubine (mg/dl) & $0.8 \pm 0.1$ & $0.7 \pm 0.1$ & 0.92 \\
\hline Alanine-transaminase $(\mathrm{U} / \mathrm{L})$ & $39.8 \pm 8.7$ & $60.1 \pm 10.3$ & 0.015 \\
\hline Gamma-glutamyl-transaminase (U/L) & $131.4 \pm 31.4$ & $127.3 \pm 47.6$ & 0.87 \\
\hline Ferritine (ng/ml) & $207.9 \pm 42.0$ & $373.5 \pm 86.4$ & 0.021 \\
\hline Albumin (mg/dl) & $4.0 \pm 0.1$ & $3.9 \pm 0.1$ & 0.83 \\
\hline Creatinine (mg/dl) & $0.9 \pm 0.1$ & $0.9 \pm 0.1$ & 0.89 \\
\hline NAFLD activity score $(0-8)$ & $0.9 \pm 0.1$ & $4.0 \pm 0.4$ & $<0.001$ \\
\hline Liver steatosis score $(0-3)$ & $0.6 \pm 0.1$ & $2.1 \pm 0.2$ & $<0.001$ \\
\hline Ballooning degeneration $(0-2)$ & $0.0 \pm 0.0$ & $0.9 \pm 0.3$ & 0.003 \\
\hline Lobular inflammation (0-3) & $0.3 \pm 0.1$ & $1.0 \pm 0.3$ & 0.031 \\
\hline Liver fibrosis $(0-4)$ & $1.1 \pm 0.2$ & $2.4 \pm 0.7$ & 0.20 \\
\hline Diagnosis of HCC $(\%)$ & 33 & 73 & - \\
\hline Preoperative LiMAx liver function $(\mu \mathrm{g} / \mathrm{kg} / \mathrm{h})$ & $424.5 \pm 24.1$ & $346.0 \pm 60.1$ & 0.36 \\
\hline
\end{tabular}

Data are given as means $\pm \mathrm{SE}$ or absolute values. Abbreviations: HbAlc, glycated hemoglobin Alc; HCC, hepatocellular carcinoma; HOMA-IR, homeostasis model assessment for insulin resistance; NAFLD, nonalcoholic fatty liver disease; NAS, NAFLD activity score. 
Lock JF (2018) Visfatin/NAMPT is unrelated to nonalcoholic fatty liver histology but correlates with liver recovery after partial liver resection: Data from a pilot study

according to HCC and non-malignant indication for liver surgery, but found comparable levels $(1.8 \pm 0.3$ vs. $2.2 \pm 0.6 ; P=0.90)$. The same was true for hepatic visfatin/NAMPT mRNA regarding all aforementioned parameters $(P>0.05$, resp., data not shown).

Univariate correlation analysis indicated absence of associations regarding measures of glucose metabolism, insulin resistance and clinical chemistry markers of liver function $(P>0.05$, resp.; Table 2). Otherwise, significant relationships with ferritine and serum iron became apparent $(P<0.05$, resp.; Table 2$)$, and trend associations of visfatin/NAMPT mRNA with white blood cell count, hepatic C-reactive protein mRNA and serum iron (Table 2).

\section{Visfatin/NAMPT and LiMAx assessed liver function}

A complete set of postoperative LiMAx data was available from $41 \%$ of included subjects. Preoperative serum visfatin/NAMPT correlated with the mean of postoperative LiMAx values $(r=0.649 ; P=0.004)$. This finding remained significant even after adjustment for liver resection volume and BMI $(P=0.044$; Figure 1$)$. Additionally, patients who were capable of recovering physiological LiMAx values within ten days after surgery had significantly elevated preoperative serum visfatin/NAMPT compared to subjects not recovering a minimum of $315 \mu \mathrm{g} / \mathrm{kg} / \mathrm{h}$ within ten days after partial hepatectomy $(P=0.014$; Figure 2$)$. Of note, the latter effect also remained significant after adjustment for BMI $(P=$ 0.026).

\section{Discussion}

Our study provides evidence that preoperative circulating visfatin/ NAMPT levels correlate with postoperative functional recovery after partial liver resection. Otherwise, our findings do not indicate that visfatin/NAMPT is associated with NAFLD histopathology or insulin resistance.

Partial hepatectomy represents a nowadays routinely performed procedure, whereby residual liver function and functional recovery strikingly impact clinical outcomes [23]. Therefore, it would be of clinical significance to identify factors capable of predicting or impacting postoperative liver regeneration. In this study we observed a significant association of preoperative serum visfatin/NAMPT with postoperative functional recovery. This observation was not directly

Table 2. Correlation studies on visfatin/NAMPT. Significant associations are shown in bold

\begin{tabular}{|c|c|c|}
\hline & $\begin{array}{l}\text { Circulating } \\
\text { visfatin/NAMPT } \\
\text { r }(P \text {-value })\end{array}$ & $\begin{array}{l}\text { Hepatic visfatin/ } \\
\text { NAMPT mRNA } \\
\text { r (P-value) }\end{array}$ \\
\hline Hepatic visfatin/NAMPT mRNA & $-0.034(0.86)$ & - \\
\hline Liver steatosis score & $0.152(0.33)$ & $0.093(0.63)$ \\
\hline Ballooning degeneration & $0.090(0.56)$ & $0.195(0.30)$ \\
\hline Lobular inflammation & $-0.023(0.88)$ & $0.201(0.29)$ \\
\hline Liver fibrosis score & $-0.002(0.99)$ & $0.287(0.12)$ \\
\hline Fasting glucose & $-0.134(0.39)$ & $0.010(0.96)$ \\
\hline HOMA-IR & $-0.055(0.72)$ & $0.062(0.75)$ \\
\hline HbA1c (\%) & $-0.079(0.62)$ & $0.293(0.14)$ \\
\hline C-reactive protein & $0.213(0.20)$ & $0.032(0.88)$ \\
\hline White blood cell count & $0.242(0.13)$ & $0.357(0.062)$ \\
\hline Hepatic C-reactive protein mRNA & $-0.120(0.44)$ & $0.346(0.061)$ \\
\hline Ferritine & $0.325(0.032)$ & $0.224(0.23)$ \\
\hline Serum iron & $-0.342(0.023)$ & $-0.323(0.082)$ \\
\hline Bilirubine & $0.042(0.79)$ & $-0.091(0.63)$ \\
\hline Alanine-transaminase & $0.102(0.51)$ & $0.166(0.38)$ \\
\hline Gamma-glutamyl-transaminase & $0.084(0.59)$ & $0.025(0.90)$ \\
\hline Aspartate-transaminase & $0.115(0.47)$ & $0.088(0.66)$ \\
\hline
\end{tabular}

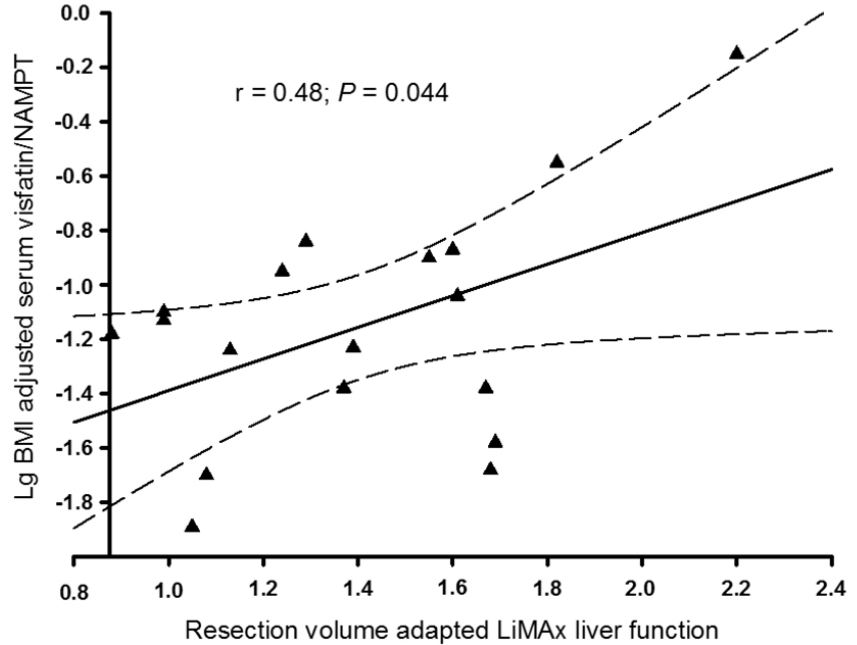

Figure 1. Serum visfatin/NAMPT and postoperative liver function

Postoperative enzymatic liver function capacity as adjusted for resected liver volume was correlated with preoperative serum visfatin/NAMPT. Lines indicate the $95 \%$ confidence intervals.

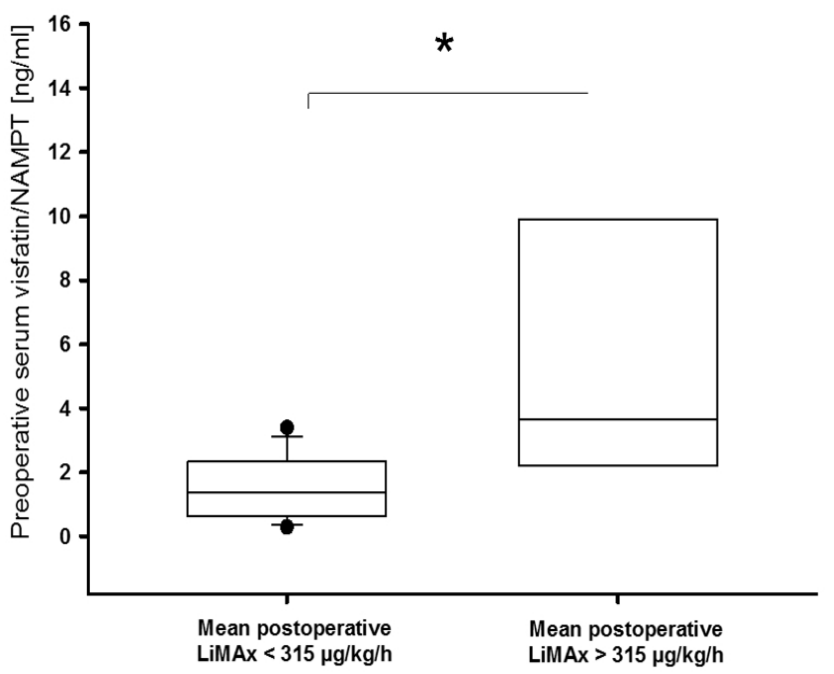

Figure 2. Elevated preoperative serum visfatin/NAMPT in subjects with recovered postoperative liver function

Subjects reaching physiological enzymatic liver capacity within ten days after surgery had higher preoperative serum visfatin/NAMPT compared to patients failing to recover physiological capacity $(P=0.014)$. Boxes span from 25 th-75th percentile, error bars indicate 10th and 90th percentile; *, $P<0.05$.

related to NASH as we found no relationship of visfatin/NAMPT with typical histopathological features, corresponding to some $[12,15,16]$ yet not all $[11,13]$ studies on visfatin/NAMPT in human NAFLD. The latter could be explained by different study populations or the use of varying assays, either detecting carboxy-terminal vs. the full length visfatin/NAMPT [26]. Our finding that visfatin/NAMPT was associated with surrogates of inflammation is otherwise consistent with its hypothesized inflammatory role $[8,9]$. We expanded the latter by our observation of a relationship of visfatin/NAMPT with surrogates of hepatic oxidative stress, namely serum iron and ferritine [27]. It has been reported in NAFLD that visfatin/NAMPT and interleukin- 6 can be co-regulated $[9,10]$. Interleukin- 6 exposure can exert antioxidative effects and improve liver regeneration after partial hepatectomy in a murine model $[28,29]$. In addition, visfatin/NAMPT was shown to have direct cell protective effects in hepatocytes [12]. Therefore, one 
Lock JF (2018) Visfatin/NAMPT is unrelated to nonalcoholic fatty liver histology but correlates with liver recovery after partial liver resection: Data from a pilot study

hypothesis to explain the observed scenario in our study could be that subjects with elevated preoperative serum visfatin/NAMPT can more rapidly respond to acute surgery induced liver injury, which could at least in part have been mediated by oxidative stress. Accordingly, our results evolve the hypothesis that visfatin/NAMPT is related to liver regeneration, but this needs to be approved in larger prospective studies.

Strengths of this study include the analysis of postoperative liver function by established methods, the use of representative liver specimens and gold standard NASH evaluation. Limitations address the inclusion of Caucasians only; and the relatively small study group. However, our data are largely in accordance with previous reports and we detected significant effects, suggesting sufficient power.

In summary we show an association of serum visfatin/NAMPT with postoperative liver regeneration.

\section{Declaration of interest}

Martin Stockmann is the inventor of the LiMAx test and has capital interest in Humedics, the company marketing the LiMAx test. None of the other authors has conflicts of interest to report.

\section{References}

1. Takahashi Y, Fukusato T (2014) Histopathology of nonalcoholic fatty liver disease/ nonalcoholic steatohepatitis. World J Gastroenterol 20: 15539-15548.

2. Zivkovic AM, German JB, Sanyal AJ (2007) Comparative review of diets for the metabolic syndrome: implications for nonalcoholic fatty liver disease. Am J Clin Nutr 86: 285-300. [Crossref]

3. Starley BQ, Calcagno CJ, Harrison SA (2010) Nonalcoholic fatty liver disease and hepatocellular carcinoma: a weighty connection. Hepatology 5: 1820-1832.

4. Fox RK (2014) When to consider liver transplant during the management of chronic liver disease. Med Clin North Am 98: 153-168. [Crossref]

5. Koskinas J, Gomatos IP, Tiniakos DG, Memos N, Boutsikou M, et al. (2008) Liver histology in ICU patients dying from sepsis: a clinico-pathological study. World $J$ Gastroenterol 14: 1389-1393.

6. Wang X, Li J, Riaz DR, Shi G, Liu C, et al. (2014) Outcomes of liver transplantation for nonalcoholic steatohepatitis: a systematic review and meta-analysis. Clin Gastroenterol Hepatol 12: 394-402.

7. Blüher M (2014) Adipokines - removing road blocks to obesity and diabetes therapy. Mol Metab 3: 230-240. [Crossref]

8. Jung UJ, Choi MS (2014) Obesity and its metabolic complications: the role of adipokines and the relationship between obesity, inflammation, insulin resistance, dyslipidemia and nonalcoholic fatty liver disease. Int J Mol Sci 15: 6184-6223.

9. Polyzos SA, Kountouras J, Mantzoros CS (2016) Adipokines in nonalcoholic fatty liver disease. Metabolism 65: 1062-1079.

10. Jarrar MH, Baranova A, Collantes R, Ranard B, Stepanova M, et al. (2008) Adipokines and cytokines in non-alcoholic fatty liver disease. Aliment Pharmacol Ther 27: 412421. [Crossref]

11. Aller R, de Luis DA, Izaola O, Sagrado MG, Conde R, et al. (2009) Influence of visfatin on histopathological changes of non-alcoholic fatty liver disease. Dig Dis Sci 54: 17721777. [Crossref]
12. Dahl TB, Haukeland JW, Yndestad A, Ranheim T, Gladhaug IP, et al. (2010) Intracellular nicotinamide phosphoribosyltransferase protects against hepatocyte apoptosis and is down-regulated in nonalcoholic fatty liver disease. J Clin Endocrinol Metab 95: 3039-3047.

13. Kukla M, Ciupinska KM, Kajor M, Wylezol M, Zwirska KK, et al. (2010) Liver visfatin expression in morbidly obese patients with nonalcoholic fatty liver disease undergoing bariatric surgery. Pol J Pathol 61: 147-153.

14. Auguet T, Terra X, Porras JA, Orellana Gavaldà JM, Martinez S, et al. (2013) Plasma visfatin levels and gene expression in morbidly obese women with associated fatty liver disease. Clin Biochem 46:202-208.

15. Genc H, Dogru T, Kara M, Tapan S, Ercin CN, et al. (2013) Association of plasma visfatin with hepatic and systemic inflammation in nonalcoholic fatty liver disease. Ann Hepatol 12: 548-55.

16. Polyzos SA, Kountouras J, Papatheodorou A, Katsiki E, Patsiaoura K, et al. (2013) Adipocytokines and cytokeratin-18 in patients with nonalcoholic fatty liver disease: Introduction of CHA index. Ann Hepatol 12: 749-757.

17. Polyzos SA, Kountouras J, Romiopoulos I, Polymerou V (2013) Serum visfatin in nonalcoholic fatty liver disease. Ann Hepatol 13: 150-151. [Crossref]

18. Döcke S, Lock JF, Birkenfeld AL, Hoppe S, Lieske S, et al. (2013) Elevated hepatic chemerin mRNA expression in human non-alcoholic fatty liver disease. Eur $J$ Endocrinol 169: 547-557. [Crossref]

19. Hoppe S, von Loeffelholz C, Lock JF, Doecke S, Sinn BV, et al. (2015) Nonalcoholic steatohepatits and liver steatosis modify partial hepatectomy recovery. J Invest Surg 2015;28: 24-31.

20. Matthews DR, Hosker JP, Rudenski AS, Naylor BA, Treacher DF, et al. (1985) Homeostasis model assessment: insulin resistance and beta-cell function from fasting plasma glucose and insulin concentrations in man. Diabetologia 28: 412-419.

21. Kleiner DE, Brunt EM, Van Natta M, Behling C, Contos MJ, et al. (2005) Design and validation of a histological scoring system for nonalcoholic fatty liver disease. Hepatology 41: 1313-1321.

22. Brunt EM, Kleiner DE, Wilson LA, Belt P, Neuschwander-Tetri BA (2011) NASH Clinical Research Network (CRN). Nonalcoholic fatty liver disease (NAFLD) activity score and the histopathologic diagnosis in NAFLD: distinct clinicopathologic meanings. Hepatology 53: 810-820.

23. Stockmann M, Lock JF, Riecke B, Heyne K, Martus P, et al. (2009) Prediction of postoperative outcome after hepatectomy with a new bedside test for maximal liver function capacity. Ann Surg 250: 119-125.

24. Lock JF, Schwabauer E, Martus P, Videv N, Pratschke J, et al. (2010) Early diagnosis of primary nonfunction and indication for reoperation after liver transplantation. Liver Transpl 16: 172-180.

25. Hector J, Schwarzloh B, Goehring J, Strate TG, Hess UF, et al. (2007) TNF-alpha alter visfatin and adiponectin levels in human fat. Horm Metab Res 39: 250-255. [Crossref]

26. Retnakaran R, Youn BS, Liu Y, Hanley AJ, Lee NS, et al. (2008) Correlation of circulating full-length visfatin (PBEF/NAMPT) with metabolic parameters in subjects with and without diabetes: a cross-sectional study. Clin Endocrinol (Oxf) 69: 885-893.

27. Nanba S, Ikeda F, Baba N, Takaguchi K, Senoh T, et al. (2016) Association of hepatic oxidative stress and iron dysregulation with HCC development after interferon therapy in chronic hepatitis C. J Clin Pathol 69: 226-233.

28. Jin X, Zimmers TA, Perez EA, Pierce RH, Zhang Z, et al. (2006) Paradoxical effects of short- and long-term interleukin-6 exposure on liver injury and repair. Hepatology 43: 474-484. [Crossref]

29. Jin X, Zhang Z, Beer-Stolz D, Zimmers TA, Koniaris LG (2007) Interleukin-6 inhibits oxidative injury and necrosis after extreme liver resection. Hepatology 46: 802-812.

Copyright: (C2018 Lock JF. This is an open-access article distributed under the terms of the Creative Commons Attribution License, which permits unrestricted use, distribution, and reproduction in any medium, provided the original author and source are credited. 